\title{
ESTIMATION OF BIRD TRAJECTORY ALONG THE FLOW FIELD OF INLET DUCT OF AN AIRCRAFT
}

\author{
Sandeep $^{1}$, K V Murlidharan ${ }^{2}$ \\ ${ }^{I} I V$ - Semester, Thermal Power Engineering, Mechanical Department, MVJ College of Engineering, Bangalore \\ ${ }^{2}$ Professor, MVJ College of Engineering, Mechanical Dept, Bangalore
}

\begin{abstract}
The momentum of the Bird projectile plays key role in the design of engine components for bird hazard and it is important to know the momentum of the bird projectile before impacting engine components. Intake duct of military aircraft have serpentine shape which increases the probability of projectile impact on duct before impacting engine components. The bird fragment at the entrance of the engine depends upon location of the bird strike and angle of impact with respect to Intake duct. There is also another probability i.e. projectile may not hit the duct at all. The aim of this project is to study the trajectory of bird projectile in the Intake-duct of a typical Military gas turbine engine. This work indicates the probability of bird hitting the duct or entering straight into the engine. Commercially available computational fluid dynamics software will be used to track the trajectory of the bird projectile. The flow field changes due to the foreign object along with the total pressure loss and distortion will be computed.
\end{abstract}

\section{INTRODUCTION}

Frontal component of an aircraft engine are vulnerable to bird strike. The component like fan rotor and blade, bullet nose, Inlet strud, inlet guide vane are designed to withstand the damage caused due to bird hit to meet safety requirement of the aircraft. It is important to know the momentum of the bird projectile before impacting engine components. The intake of the engine will have an effect on bird trajectory. It is important to know the flow physics involved inside the duct. According to the Colin James Fiola [1], the far filed boundary condition can be mentioned while performing a simulation along with pressure outlet and smooth wall. Flow physics was also mentioned and the there was a study done on turbulence model which shows that Transition SST models predicted the best results. This was evident by the Prasath MS et al in there research work [2][3].

This is an unsteady simulation having a moving mesh with six degree of freedom. To simulate this an CFD tool was used which is less time consuming and very cheap way to fulfill our needs of knowing the probability of bird hitting the walls of an aircraft duct. This is a problem having load on the bird due to its self-weight, gravity pull, its inertia forces and the forces acting on it due to the fluid flowing around it. In this report we will see how to account for this problem and setup a method to find the trajectory of the bird flowing inside an aircraft intake duct.

\section{METHODOLOGY}

To start with this CFD approach, it is an important to know the type of duct and about the moving bird. To carry out the simulation, a serpentine type (S-type) intake duct of an aircraft was used. Flow domain of intake duct was modeled in SOLIDWORKS tool. The mesh was generated using the CD-adapco's CFD tool named as StarCCM+. The Trimmed mesh model was used which is having trimmed hexahedral cell shape based core mesh. The quality of mesh was checked and the prism layers were defined so as to consider the flow in the boundary layers. The mesh was refined in the duct and near its inlet region using the volume control parameters. Than the study state analysis was performed on the duct using a eulerian approach. In this case the flow is turbulent and compressible. The Reynolds Averaged NavierStokes equations were used to estimate the turbulent flow inside the intake duct with the SST k- $\omega$ turbulence model. The Coupled Flow Model is used together with threedimensional model. Gas was selected as a fluid material and corresponding equation of state was selected as Ideal gas. The boundaries were defined as freestream, wall and outlet. At the freestream, the flight Mach number was defined as the boundary parameters along with the flow direction. Also the static values of pressure and temperature were defined at freestream. At the outlet, static pressure along with static temperature was defined as the boundary parameters. For the convergence monitor plots was utilized i.e. Residual plot, Pressure recovery plot at the AIP and the Outlet Mass Flow Rate plot. The validation test case was simulated on the NACA-type S-duct inlet geometry [4]. The comparison of data obtained from the experiment and the data obtained from the simulation ran on CD-adapco STAR-CCM+ tool was plotted and found a close match between two.

Further an unsteady simulation was performed where the moving bird was considered in the flow domain. The bird was simulated using a dynamic overset mesh thrown in the flow field of the intake duct. The overset mesh was assigned to a 6-DOF motion solver and the bird density along with its forces was defined in the simulation setup. The Implicit Unsteady time model was used and the Lagrangian eularian approach was used to simulate the trajectory of the bird. To start with the unsteady simulation, first the simulation was iterated as a steady stare simulation. When the flow gets converge, the unsteady simulation gets started 
with its first time step iteration. This simulation was considered as a first set of results to setup the unsteady state simulation.

Based on time step, inner iteration for each time step and mesh density parameters, the parameters study was performed. The best suited parameter was found to be having a time step of $0.0005 \mathrm{sec}$ with 20 number of inner iteration with mesh density of 2.4 million cells. Further the simulations were performed on different bird position and its orientation. The simulation stops when the bird reaches exit of the duct or else when it reaches to the wall hand hits it. In the results for all the cases, the point of hit was found out. The outlet mass flow rate, pressure recovery at the AIP and the bird translation along with its velocity and acceleration are plotted for each unsteady simulation. The flow distortion was also shown along the flow field using a Mach number counter plot.

\section{DESCRIPTION}

The simulation was performed and the results were obtained for all the cases. Hear the results are shown for the case run of bird positioned at the center with its axis parallel to the $\mathrm{x}$ axis. The mesh size was 2.4 million cells. The time step was $0.0005 \mathrm{sec}$ with 20 number of inner iteration per time step. The flow filed changes are shown in figure 1 using Mach number contour plot. The position of bird and wall interaction is also shown. The other results are plotted in figure 2 such as Absolute Total Pressure Recovery at the exit to the inlet of the duct, Outlet mass flow rate, Translation and Velocities along all the three arises. The results obtained in the validation of NACA type S-duct inlet is shown in figure 3 . The results obtained from the simulation and the experimental results obtained from the AGARD Advisory Report 270 is plotted in the graph which shows a close match between two.

\subsection{Sketches/Graphs}

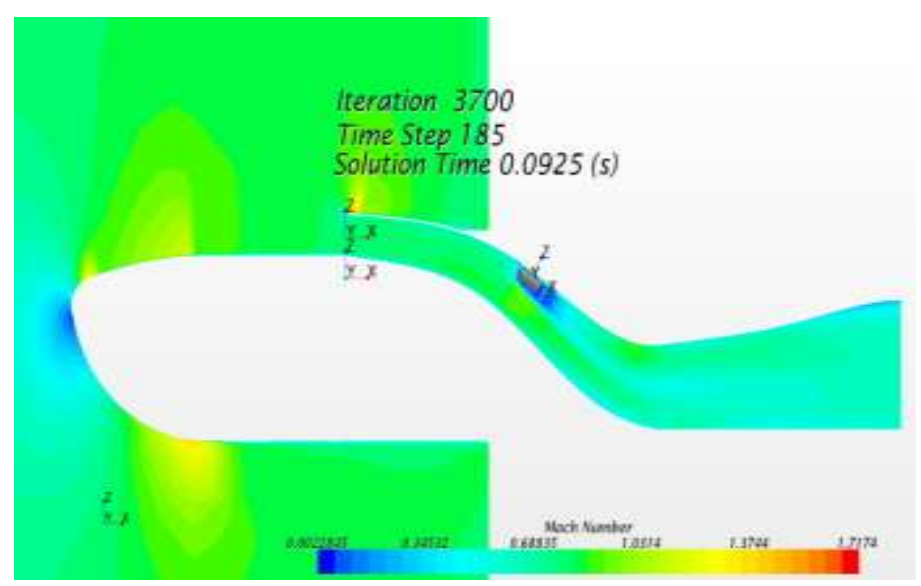

Fig 1: The Mach number contour plot with the Bird's point of hit.

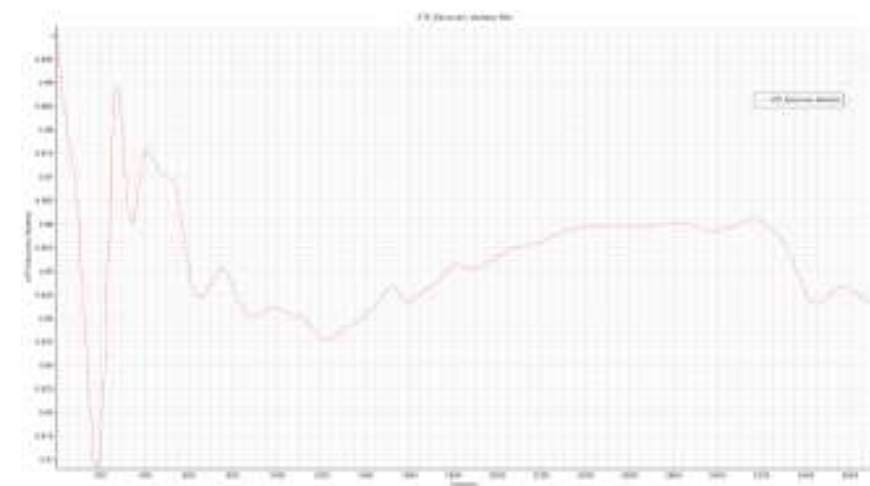

(a)

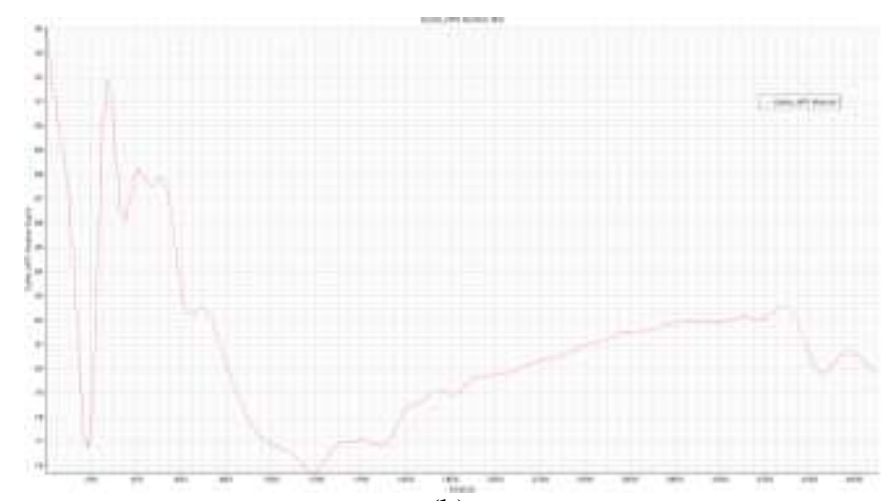

(b)

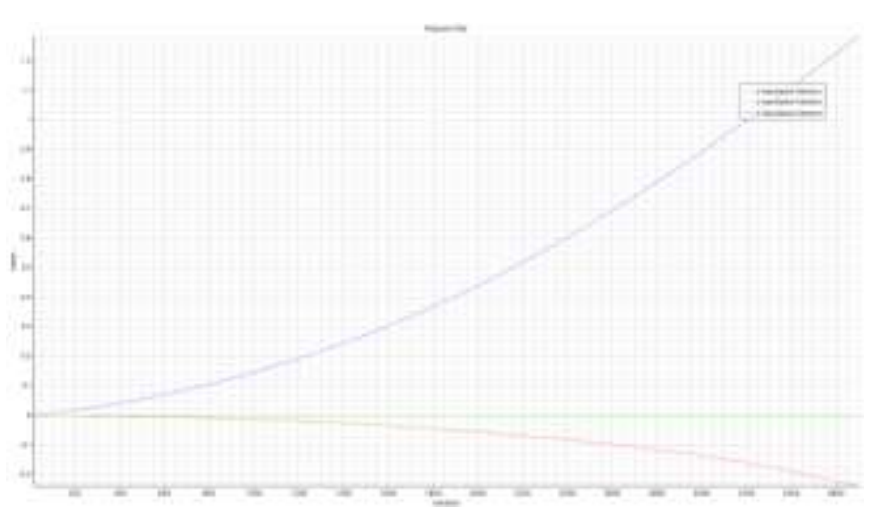

(c)

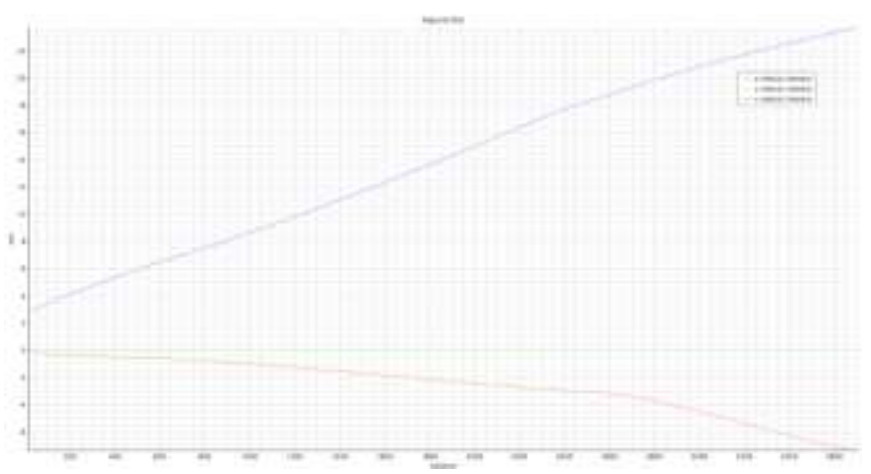

(d)

Fig 2: Plots of (a) ATP recovery at AIP, (b) Mass Flow Rate, (c) Translation and (d) Velocity 


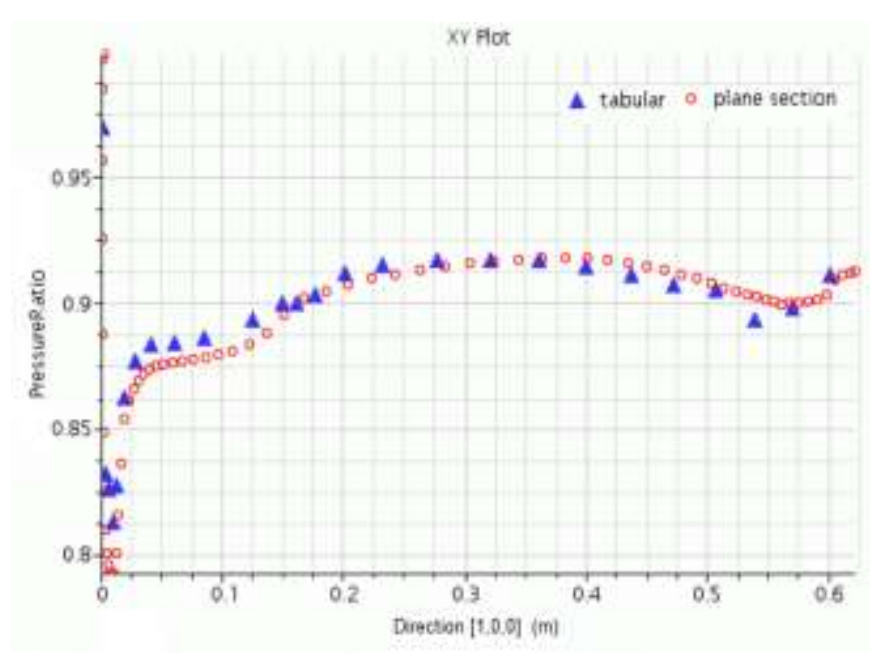

Fig 3: Plot displays an external data set consisting of experimental data together with the simulation data.

\section{CONCLUSION}

The trajectory of the moving bird inside an aircraft intake duct was simulated successfully for various Mach numbers, bird position and its orientation. The results for one simulating condition are shown in the appendix. The results contains the location at which the bird hit, change in the flow field at the point of hit and pressure recovery at the AIP. The bird translation, velocity and acceleration are also plotted along all the three axis of the global coordinate system.

It was observed using the results of the simulations that the bird have a high momentum while entering into the duct and due to its inertia it does not follow the stream line of the flow path and hits the duct before reaching to the duct exit. It is expected that due to the momentum of the bird at the point of hit, the bird will get fragmented before reaching to the duct exit. The probability of bird hitting the duct was found high as it was found that all the simulation had the bird collision with the duct wall. There was no simulation that shows the bird getting injected into the duct and reaches to the exit without hitting the duct wall. With this it can be concluded that bird probability to reach to the exit is very low.

\section{REFERENCES}

[1] Colin James Fiola. "Numerical Simulation Of Separated And Secondary Flows In Diffusing S-Ducts For Air Breathing Propulsion". THESIS / Master of Science in Aerospace Engineering, Washington University in St. Louis/August 2013.

[2] Prasath MS, Shiva Shankar Gowda AS, Senthilkumar S. "CFD Study of Air Intake Duct". IJESR/May 2014/ Vol-4/Issue-5/282-288.

[3] Prasath MS, Shiva Shankar Gowda AS, Senthilkumar S. "CFD Study of Air Intake Diffuser". IJESR/2014/Volume-3 /Issue-01 /Pages 53-59.
[4] "Air Intakes for High Speed Vehicles," AGARD-AR270 (AGARD Advisory Report 270), Fluid Dynamics Panel Working Group 13, September, 1991. 